\title{
Serine palmitoyltransferase long chain subunit 3 is associated with hepatocellular carcinoma in patients with NAFLD
}

\author{
SHO IJUIN $^{1}$, KOHEI ODA $^{1}$, SEIICHI MAWATARI $^{1}$, OHKI TANIYAMA $^{1}$, AI TOYODOME $^{1}$, \\ HARUKA SAKAE $^{1}$, KAZUAKI TABU ${ }^{1}$, KOTARO KUMAGAI ${ }^{1}$, SHUJI KANMURA ${ }^{1}$, \\ TSUTOMU TAMAI ${ }^{2}$, AKIHIRO MORIUCHI $^{3}$, HIROFUMI UTO $^{4}$ and AKIO IDO ${ }^{1}$
}

\author{
${ }^{1}$ Digestive and Lifestyle Diseases, Kagoshima University Graduate School of Medical and Dental Sciences, \\ Kagoshima 890-8520; ${ }^{2}$ Department of Gastroenterology and Hepatology, Kagoshima City Hospital, Kagoshima 890-8760; \\ ${ }^{3}$ Department of Gastroenterology, National Hospital Organization Kagoshima Medical Center, Kagoshima 892-0853; \\ ${ }^{4}$ Center for Digestive and Liver Diseases, Miyazaki Medical Center Hospital, Miyazaki 880-0003, Japan
}

Received June 2, 2021; Accepted October 14, 2021

DOI: $10.3892 / \mathrm{mco} .2021 .2488$

\begin{abstract}
The prevalence of non-alcoholic fatty liver disease (NAFLD) is continuously increasing, with the proportion of patients with liver carcinogenesis due to non-alcoholic steatohepatitis (NASH) rising accordingly. Although it is important to identify individuals with hepatic carcinogenesis among patients with NAFLD, useful biomarkers have not yet been established. Previously, in a mouse model of diabetes mellitus without genetic modifications, we reported that a high-fat diet increases serine palmitoyltransferase long chain subunit 3 (SPTLC3) expression in liver tissue, accompanied by high frequency of liver carcinogenesis. Serine palmitoyltransferase (SPT) catalyzes the metabolism of fatty acids, particularly sphingolipid synthesis, and SPTLC3 has been identified as its catalytic subunit, but its role in liver disease is unclear. In the present study, the importance of SPTLC3 in NAFLD development was investigated. SPTLC3 mRNA expression was observed in a liver cancer cell line and in liver tissues from patients with NAFLD and liver cancer. In total, 99 patients with NAFLD (66 without hepatocellular carcinoma (HCC) and 33 with HCC were recruited, having been diagnosed by liver biopsy or imaging, along with 6 healthy volunteers (HVs).
\end{abstract}

Correspondence to: Dr Kohei Oda, Digestive and Lifestyle Diseases, Kagoshima University Graduate School of Medical and Dental Sciences, 8-35-1 Sakuragaoka, Kagoshima 890-8520, Japan E-mail: odakou@m2.kufm.kagoshima-u.ac.jp

Abbreviations: SPT, serine palmitoyltransferase; SPTLC3, serine palmitoyltransferase long chain subunit 3; NASH, non-alcoholic steatohepatitis; NAFLD, non-alcoholic fatty liver disease; ALD, alcoholic disease; $\mathrm{HBV}$, hepatitis $\mathrm{B}$ virus; $\mathrm{HCV}$, hepatitis $\mathrm{C}$ virus; $\mathrm{HCC}$, hepatocellular carcinoma; HV, healthy volunteers; ALT, alanine aminotransferase; LDL-Chol, low-density lipoprotein cholesterol; AFP, $\alpha$-fetoprotein; DCP, des- $\gamma$-carboxy prothrombin

Key words: SPTLC3, NAFLD, NASH, HCC, liver fibrosis
Serum was collected from patients and HVs, and SPTLC3 level was assessed by ELISA. SPTLC3 expression was higher in non-cancerous compared with that in cancerous liver tissues. Serum SPTLC3 levels were negatively correlated with platelet count and positively correlated with hyaluronic acid levels, suggesting an association with liver fibrosis. Moreover, SPTLC3 levels were significantly higher in the HCC group than in the HV and NAFLD groups. Multivariate analysis of HCC-related factors identified platelets, alanine transferase, albumin and SPTLC3 as independent factors associated with HCC. Furthermore, in patients with other chronic liver diseases (hepatitis B and C, and alcoholic liver disease), no significant differences in serum SPTLC3 levels were observed between patients with or without HCC. Thus, SPTLC3 expression increases specifically with the progression of NAFLD. Overall, the present results indicate that SPTLC3 may be involved in the development of liver carcinogenesis during NAFLD.

\section{Introduction}

Non-alcoholic fatty liver disease (NAFLD) is a condition in which fatty liver is diagnosed histologically or using imaging in the absence of other liver diseases, such as alcoholic liver disease (ALD). Considering that the onset of NAFLD is a consequence of obesity, diabetes, dyslipidemia, hypertension or other metabolic disorders, it is currently considered as a liver phenotype of metabolic syndrome, and, in recent years, a concept known as metabolic-associated fatty liver disease (MAFLD) has been proposed (1).

NAFLD progression is histologically characterized by large hepatic lipid droplets and is classified into non-alcoholic fatty liver, in which the condition hardly progresses, and non-alcoholic steatohepatitis (NASH), in which the condition progresses and may subsequently lead to cirrhosis and liver cancer.

The global prevalence of NAFLD increased from $20.1 \%$ in 2000-2005 to 23.8\% in 2006-2010 and 26.8\% in 2011-2015 (2). Changes in the prevalence of NASH are not fully known, but it is thought to increase in parallel with an increase in NAFLD 
prevalence. In Japan, there were 660,000 patients with NAFLD and advanced fibrosis of stage 3 or higher in 2016, and this is expected to increase to 990,000 by 2030 (3).

The rate of liver carcinogenesis from NAFLD is as low as $0.44 / 1,000$ person-years (2); however, the risk reportedly increases with the progression of liver pathology to 5.29/1,000 person-years for NASH and 20/1,000 person-years for cirrhosis in Japan (4). Although this is low compared to the carcinogenesis rate from other liver diseases, as aforementioned, the prevalence of NAFLD is extremely high, and a Japanese nationwide survey on patients with hepatocellular carcinoma (HCC) revealed that the proportion of patients with non-viral etiologies, including NAFLD, had increased from $10.0 \%$ in 1991 to $32.5 \%$ in 2015, and is continually increasing (5).

Regarding prognosis, liver disease-related mortality in patients with NAFLD increases with the progression of liver fibrosis (6), with liver fibrosis being reported as the factor most significantly associated with prognosis among other pathological findings in NAFLD (7).

Serine palmitoyltransferase (SPT) catalyzes fatty acid metabolism, particularly sphingolipid synthesis, and serine palmitoyltransferase long chain base subunit 3 (SPTLC3) has recently been identified as its catalytic subunit; however, its association with and contribution to liver disease remain unclear. We previously demonstrated that SPTLC3 was highly expressed in the liver tissue in a mouse model of NASH, which frequently displays hepatocellular carcinoma (HCC) (8).

Herein, the present study aimed to analyze the association between SPTLC3 and NAFLD pathological progression, as well as liver carcinogenesis, by examining SPTLC3 expression in human liver cancer cell lines and human serum/liver tissues.

\section{Materials and methods}

Patients and sample collection. In total, 99 patients diagnosed with NAFLD (66 without HCC and 33 with HCC) and 6 healthy volunteers (HVs) were recruited at the Digestive and Lifestyle Diseases, Kagoshima University Graduate School of Medical and Dental Sciences between August 2016 and June 2020. The patient population consisted of 27 men and 62 women with a median age of 58 years (range, 22-86 years). NAFLD was diagnosed based on the clinical guidelines of the American Association for the Study of Liver Diseases (9) and The European Association for the Study of the Liver (10). Patient inclusion criteria were as follows: i) 5\% or more of liver cells containing lipid droplets detected by liver biopsy, or evidence of fatty liver on using ultrasound (US) or computed tomography (CT) imaging; and ii) daily alcohol intake of $<30 \mathrm{~g}$ for men and $<20 \mathrm{~g}$ for women and negativity for hepatitis virus markers to exclude viral liver disease and autoimmune liver diseases. Those who met both i) and ii) were diagnosed with NAFLD $(9,10)$. The diagnosis of HCC was made by a radiologist and a hepatologist using contrast-enhanced CT or contrast-enhanced MRI. Subjects were considered as HVs if they had no history of lifestyle-related diseases, including NAFLD. Serum was collected from both the NAFL/NASH groups (without HCC) and the HCC group on the day of the first visit to the Digestive and Lifestyle Diseases, in the blood laboratory of the Kagoshima University Graduate School of
Medical and Dental Sciences, on the day before the biopsy and before the intervention, in the period between August 2016 and June 2020, in order to evaluate SPTLC3 concentration using ELISA, and the levels of blood biochemical parameters [platelet count, aspartate aminotransferase (AST), alanine aminotransferase (ALT), $\gamma$-glutamyl transferase, total bilirubin, albumin, prothrombin index, low-density lipoprotein cholesterol (LDL-Chol), triglyceride, glucose, HbA1c, hyaluronic acid, $\alpha$-fetoprotein (AFP) and des- $\gamma$-carboxy prothrombin (DCP)] were also analyzed. Blood biochemical parameters were evaluated via routine laboratory tests that were conducted at the same time as the SPTLC3 assay. Furthermore, as a combination of clinical and routine laboratory parameters of liver fibrosis, fibrosis index based on the four factors (FIB-4 index) [age (year) x AST (U/1)/ pplatelet count $\left(1 \times 10^{9} / 1\right) \times \sqrt{ }$ ALT (U/1)\}] (11-13) and AST to platelet ratio index (APRI) [AST/upper limit of normal x 100)/platelet count] were calculated based on the aforementioned blood biochemical parameters $(13,14)$. Non-tumor and tumor parts were collected from the surgical specimens of patients with NAFLD and HCC.

In addition to the patients mentioned earlier, serum SPTLC3 levels were measured in 31 patients with hepatitis B virus (HBV), 36 patients with hepatitis $\mathrm{C}$ virus (HCV) and 24 patients with alcoholic liver disease (ALD). Patients positive for serum HBs antigen were considered to have HBV; those positive for $\mathrm{HCV}$ antibody were considered to have $\mathrm{HCV}$; and those with daily alcohol intake $>60 \mathrm{~g}$ were classed as patients with ALD. The patients with HBV, HCV and ALD consisted of patients both with and without HCC at the time of the SPTLC3 assay. In the present study, the SPTLC3 values were compared between both groups.

The present study was approved by the Ethics Committee of Kagoshima University Hospital (approval no. 28-107) and written informed consent was obtained from all the patients. All procedures were performed in accordance with the World Medical Association's Declaration of Helsinki.

Reverse transcription-quantitative (RT-q)PCR. SPTLC3 expression levels were assessed in human liver cancer cell lines and human liver tissues. HepG2, Huh7 and Hep3B cells (obtained from Sumitomo Dainippon Pharma Co., Ltd.) were selected as the liver cancer cell lines; HT29 and HCT116 cells (obtained from DS Pharma Biomedical Co., Ltd.) were selected as the colorectal cancer cell lines; and Panc1 cells (obtained from DS Pharma Biomedical Co., Ltd.) were selected as the pancreatic cancer cell line. Regarding the liver tissues, non-tumor and tumor sections were collected from the surgical specimens of patients with NAFLD and HCC, as aforementioned. Total RNA was extracted from cells and liver tissues using the TRIzol ${ }^{\circledR}$ reagent (Thermo Fisher Scientific, Inc.). RNA purity was confirmed by spectrophotometry, and A260/A280 ratios ranged from 1.9 to 2.1. First-strand cDNA was synthesized from $500 \mathrm{ng}$ of total RNA using a PrimeScript RT Master Mix (Takara Bio Inc.) according to the manufacturer's protocol. Real-time PCR was performed using TB Green Premix Ex Taq II (Takara Bio Inc.) and the ABI Prism 7700 sequence detection system (Applied Biosystems; Thermo Fisher Scientific, Inc.). Data were collected and analyzed using the Step One Plus Real-Time PCR System 
Table I. Oligonucleotide sequence of primers for quantitative reverse transcription-quantitative PCR.

\begin{tabular}{lll}
\hline Genes & Forward primer $\left(5^{\prime} \rightarrow 3^{\prime}\right)$ & Reverse primer $\left(5^{\prime} \rightarrow 3^{\prime}\right)$ \\
\hline$S P T L C 3$ & GCTCGGTTTTGTGTTTCAGCGG & TGCCGGGAATATTTCAGTTGCAAG \\
$A C T B$ & TGGCACCCAGCACAATGAA & CTAAGTCATAGTCCGCCTAGAAGCA
\end{tabular}

SPTLC3, serine palmitoyltransferase long chain subunit 3; ACTB, $\beta$-actin.

(Applied Biosystems; Thermo Fisher Scientific, Inc.). Relative gene expression values were calculated using the comparative $\Delta \Delta \mathrm{Cq}$ method (15) and the $\mathrm{Cq}$ values were normalized to those of $\beta$-actin. The PCR conditions were as follows: Initial holding period at $95^{\circ} \mathrm{C}$ for $30 \mathrm{sec}$, followed by 40 cycles of a two-step program consisting of denaturation at $95^{\circ} \mathrm{C}$ for $5 \mathrm{sec}$ and annealing, and polymerization at $60^{\circ} \mathrm{C}$ for $34 \mathrm{sec}(16)$. The primer sequences used in this study are provided in Table I.

ELISA. SPTLC3 levels were assessed in the serum from patients with NAFLD and HVs using the ELISA kit For Serine Palmitoyltransferase, Long Chain Base Subunit 3 (SPTLC3) from Cloud-Clone Corp. (cat. no. SEH136Hu) according to the manufacturer's protocol.

Statistical analysis. Results are presented as the mean or median. At least two repeated experiments were conducted. Statistical analyses were performed using IBM SPSS version 23 (IBM Corp.). For the test method, Fisher's exact test, Mann-Whitney U test, Wilcoxon's signed-rank test, Spearman's rank correlation coefficient and Tukey's HSD test were used. All possible models of binomial logistic regression analysis were used for the multivariate analysis. Items that were significant in the univariate analysis were used as reference for the selection of possible variables. Data were considered statistically significant when $\mathrm{P}<0.05$.

\section{Results}

Human liver cancer cell lines express high levels of SPTLC3. The expression of SPTLC3 was investigated in cancer cell lines and found to be higher in HepG2, Huh7 and Hep3B cells compared that in cell lines derived from the colon, rectum and pancreas (Fig. 1).

SPTLC3 is highly expressed in non-tumor liver tissues in patients with NAFLD. Subsequently, the difference in expression level of SPTLC3 was compared between non-tumor and tumor regions of the liver in NAFLD patients with HCC. The expression level was significantly higher in the non-tumor sections compared with that in the tumor sections of the liver ( $\mathrm{P}=0.034$; Fig. 2).

Comparison of clinical characteristics between the NAFL/NASH and HCC groups. The association between the clinical characteristics and SPTLC3 expression in patients with NAFL/NASH was investigated. The results of the biochemical tests performed in the NAFL/NASH group without HCC and the group with HCC are shown in Table II. Compared with

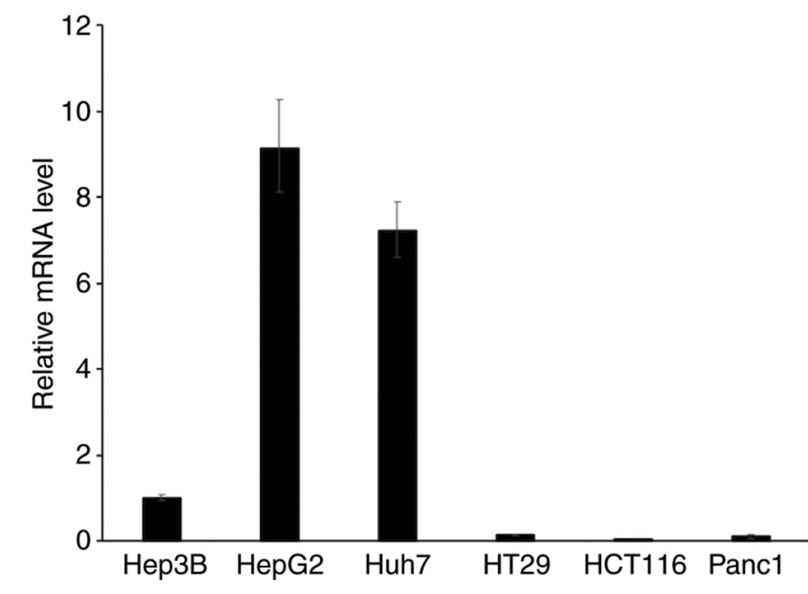

Figure 1. SPTLC3 $m R N A$ levels of six cell lines assessed by reverse transcription-quantitative PCR. Data are presented as the mean \pm SD $(n=6$ in each group). SPTLC3, serine palmitoyltransferase long chain subunit 3 .

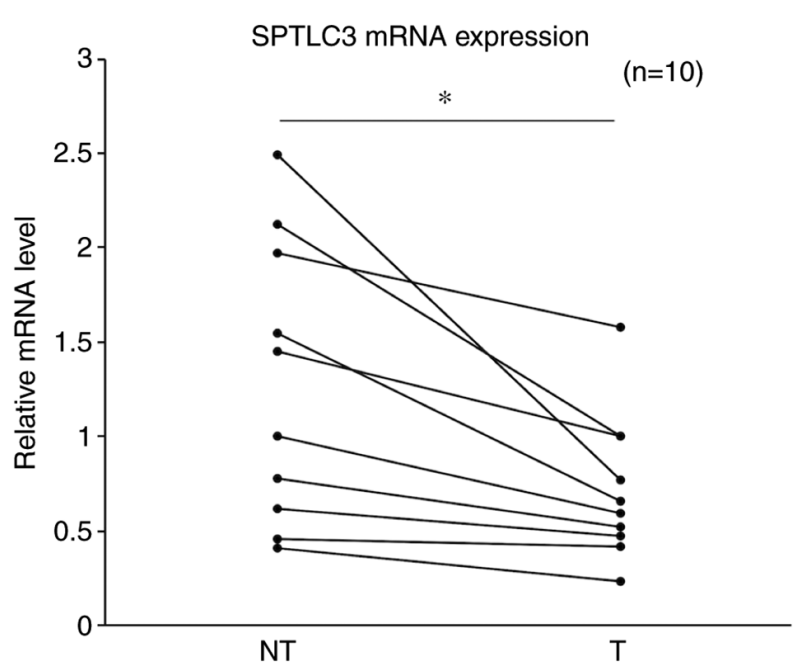

Figure 2. SPTLC3 mRNA expression in tumor and non-tumor areas of liver tissues in 10 patients with non-alcoholic fatty liver disease-related hepatocellular carcinoma. Statistical analyses were performed using Wilcoxon's signed-rank test. ${ }^{*} \mathrm{P}<0.05$. NT, non-tumor area; T, tumor area; SPTLC3, serine palmitoyltransferase long chain subunit 3 .

the NAFL/NASH group, the HCC group had lower platelet count, ALT, albumin, triglyceride and LDL-Chol levels. The patients were also older and exhibited higher HbAlc and hyaluronic acid levels. The levels of AFP and DCP, which are liver tumor markers, were significantly elevated in the HCC group $(\mathrm{P}<0.001)$. Notably, SPTLC3 levels were significantly higher in the HCC group $(\mathrm{P}=0.001$; Table II). 
Table II. Clinical characteristics of patients with NAFLD without vs. with HCC.

\begin{tabular}{|c|c|c|c|}
\hline Characteristics & NAFL/NASH (n=66) & $\mathrm{HCC}(\mathrm{n}=33)$ & P-value \\
\hline Sex (male/female) & $24 / 42$ & $13 / 20$ & 0.827 \\
\hline Age (years) & 51 & 70 & $<0.001$ \\
\hline Platelet count $\left(1 \times 10^{4} / \mu 1\right)$ & 23.9 & 12.9 & $<0.001$ \\
\hline Aspartate aminotransferase (U/l) & 71 & 39 & $<0.001$ \\
\hline Alanine aminotransferase (U/1) & 112.5 & 26 & $<0.001$ \\
\hline$\gamma$-Glutamyl transferase $(\mathrm{U} / \mathrm{l})$ & 65.5 & 58 & 0.456 \\
\hline Total bilirubin (mg/dl) & 0.7 & 0.7 & 0.278 \\
\hline Albumin $(\mathrm{g} / \mathrm{dl})$ & 4.5 & 3.8 & $<0.001$ \\
\hline Prothrombin index $(\%)$ & 105 & 99 & 0.116 \\
\hline LDL cholesterol (mg/dl) & 120 & 96 & 0.003 \\
\hline Triglyceride (mg/dl) & 128 & 114 & 0.037 \\
\hline Glucose (mg/dl) & 99 & 107 & 0.183 \\
\hline $\mathrm{HbA} 1 \mathrm{c}(\%)$ & 6 & 6.5 & 0.043 \\
\hline Hyaluronic acid level (ng/ml) & 35.3 & 181.1 & $<0.001$ \\
\hline$\alpha$-fetoprotein $(\mathrm{ng} / \mathrm{ml})$ & 2.7 & 10 & $<0.001$ \\
\hline $\mathrm{DCP}(\mathrm{mAU} / \mathrm{ml})$ & 23 & 42 & $<0.001$ \\
\hline SPTLC3 (ng/ml) & 0.935 & 1.343 & 0.001 \\
\hline FIB-4 index & 1.3775 & 3.8333 & $<0.001$ \\
\hline APRI & 0.9999 & 0.9649 & 0.812 \\
\hline HCC stage (I/II/III/IV) & - & $7 / 12 / 14 / 0$ & - \\
\hline Tumor vascularity (hyper/hypo) & - & $30 / 3$ & - \\
\hline
\end{tabular}

Data are presented as medians or n-values. Statistical analyses were performed using Mann-Whitney U test and Fisher's exact test. NAFL, non-alcoholic fatty liver; NASH, non-alcoholic steatohepatitis; NAFLD, non-alcoholic fatty liver disease; HCC, hepatocellular carcinoma; LDL, low-density lipoprotein; DCP, des- $\gamma$-carboxy prothrombin; SPTLC3, serine palmitoyltransferase long chain subunit 3 ; FIB-4 index, fibrosis index based on the four factors; APRI, aspartate aminotransferase to platelet ratio index.

SPTLC3 is associated with advanced liver fibrosis. The association of platelet count and hyaluronic acid levels (which are serum biomarkers of liver fibrosis) with SPTLC3 expression were subsequently investigated. Serum SPTLC3 exhibited a significant negative correlation with platelet count $(\mathrm{P}=0.008)$ and a significant positive correlation with hyaluronic acid levels ( $\mathrm{P}=0.007$; Fig. 3). Conversely, no significant correlations were found with AFP or DCP levels, which are biomarkers of HCC. Furthermore, FIB-4 index and APRI, which are a combination of laboratory parameters indicating liver fibrosis, were assessed. SPTLC3 expression level exhibited a significant positive correlation with FIB4-index ( $\mathrm{P}=0.007$; Fig. 3).

SPTLC3 levels are significantly higher in patients with HCC. Next, it was verified whether serum SPTLC3 levels increase during the process of carcinogenesis. SPTLC3 serum levels of the HV, NAFL/NASH and HCC groups were compared (Fig. 4). SPTLC3 levels were significantly higher in the HCC group compared with those in the HV and NAFL/NASH groups $(\mathrm{P}<0.01)$.

SPTLC3 is associated with HCC. Subsequently, multivariate statistical analysis was performed to determine whether serum SPTLC3 level was associated with carcinogenesis. Multivariate analysis of HCC-related factors was conducted using 11 factors that were significantly higher $(\mathrm{P}<0.05)$ in the HCC group following the univariate analysis; these revealed that platelet COUNT, ALT, albumin and SPTLC3 levels were independent factors associated with HCC (Table III).

SPTLC3 is significantly elevated in liver cancer specifically during NAFLD. SPTLC3 serum levels in patients with liver disease of different etiologies were compared. Among patients with HBV, HCV and alcoholic liver injury, the serum SPTLC3 levels of the non-HCC and HCC groups were compared, but no significant increase was observed in the HCC group $(\mathrm{P}>0.05$; Fig. 5). Thus, it was confirmed that SPTLC3 levels increased significantly only in the NAFLD patients with HCC compared with those without HCC.

\section{Discussion}

In the present study, SPTLC3 mRNA was found to be highly expressed in human liver cancer cell lines. In addition, SPTLC3 was associated with the development of liver fibrosis in NASH. Furthermore, serum SPTLC3 levels in patients with liver cancer and NAFLD were significantly higher compared with those in non-cancer-bearing patients.

Tabassum et al (17) performed genome-wide association analyses of 141 lipid species, followed by phenome-wide scans with 25 cardiovascular disease-related phenotypes. They identified two variants near SPTLC3 and ZNF385D that 

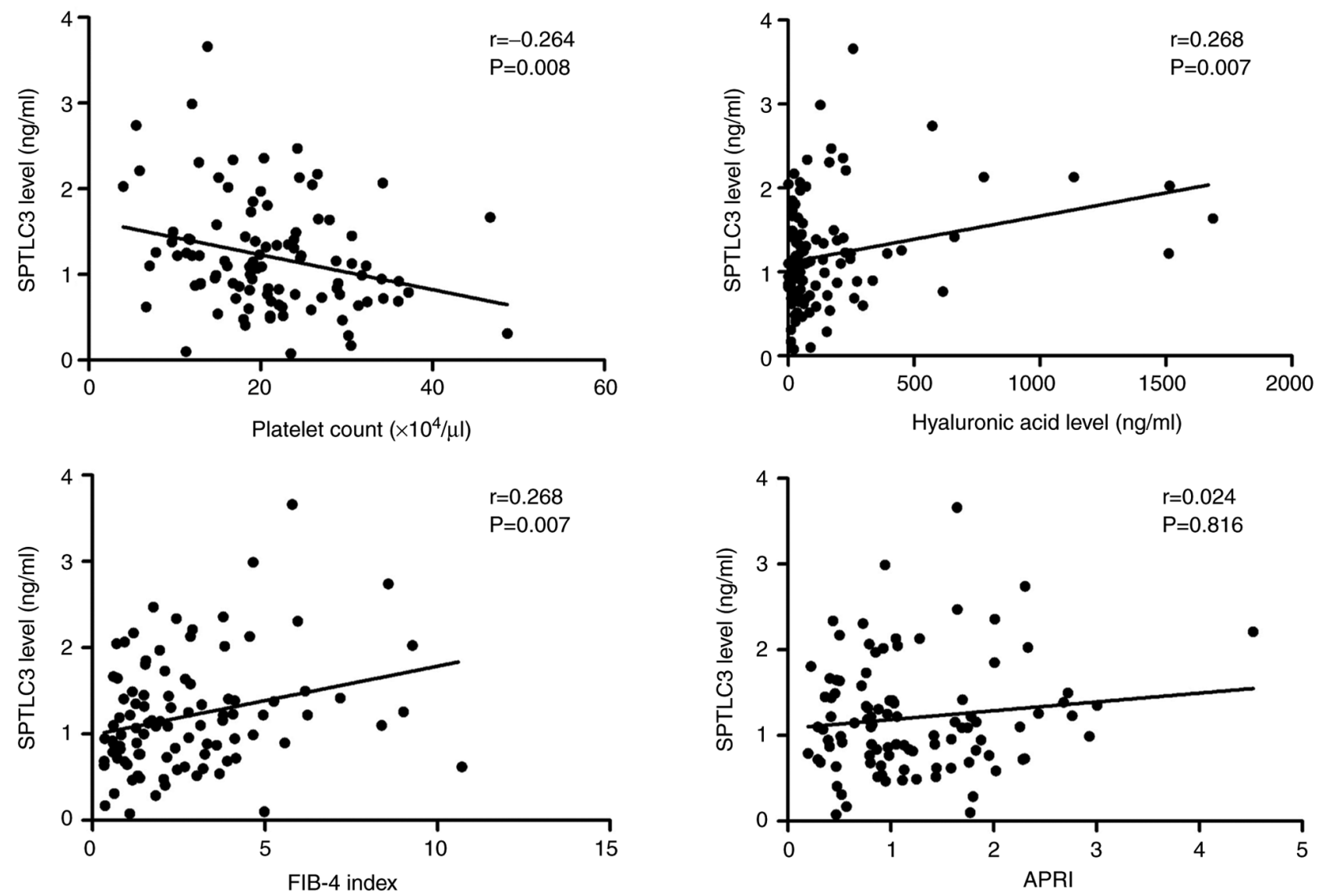

Figure 3. Correlation between SPTLC3 expression and platelet count, hyaluronic acid levels, FIB-4 index and APRI. Hyaluronic acid is plotted on a logarithmic axis. Statistical analyses were performed using Spearman's rank correlation coefficient. SPTLC3 was negatively correlated with platelet count and positively correlated with hyaluronic acid levels and FIB-4 index, suggesting an association with liver fibrosis. SPTLC3, serine palmitoyltransferase long chain subunit 3; FIB-4 index, fibrosis index based on the four factors; APRI, aspartate aminotransferase to platelet ratio index.

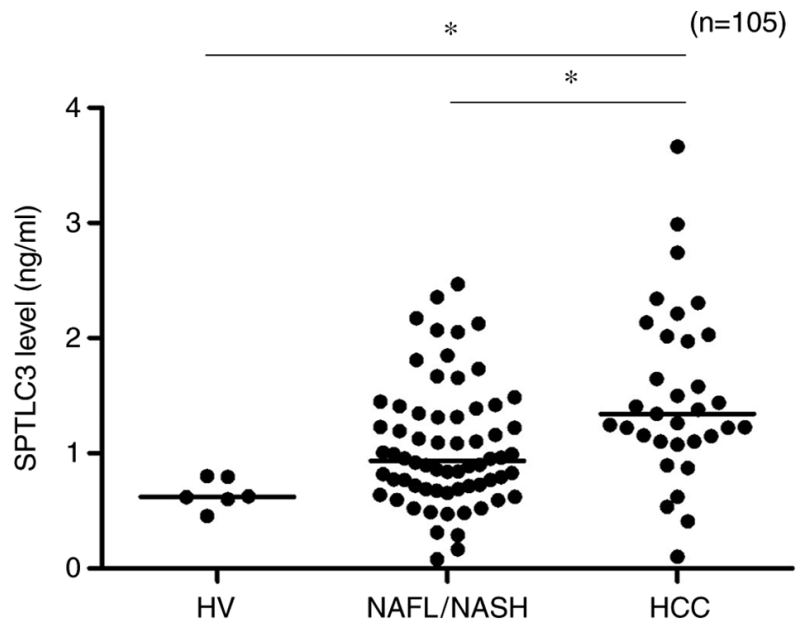

Figure 4. Serum SPTLC3 levels in HVs, patients with NAFLD without HCC and patients with both NAFLD and HCC. The horizontal lines in the dot plot represent the median. Statistical analyses were performed using Tukey's post hoc test and the Kruskal-Wallis test. "P<0.01. SPTLC3, serine palmitoyltransferase long chain subunit 3; HVs, healthy volunteers; NAFL, non-alcoholic fatty liver; NASH, non-alcoholic steatohepatitis; HCC, hepatocellular carcinoma.

modulate the plasma levels of ceramide (CER) d18:1/24:1 and d18:1/24:0, respectively, and this was associated with the risk of intracerebral hemorrhage. Therefore, SPTLC3 has been attracting attention as a lipid-related gene that may predict cardiovascular disease risk.

SPT is a membrane-bound protein localized in the endoplasmic reticulum membrane in eukaryotes. It is a complex with a molecular weight of $480 \mathrm{kDa}$, composed of three different subunits (SPTLC1, SPTLC2 and SPTLC3). SPT as a whole is composed of four dimers, including two subtypes of dimers: SPTLC1 and SPTLC2; and SPTLC1 and SPTLC3. The N-terminus of SPTLC1 binds to the endoplasmic reticulum membrane, while the C-terminus binds to the paired SPTLC 2 or SPTLC3 in the cytoplasm and exhibits enzymatic activity (18). Notably, silencing SPTLC3 expression in HepG2 or human trophoblast cells using SPTLC3-specific siRNA significantly attenuates intracellular SPT activity. This suggests that SPTLC3 represents a key subunit of SPT activity in liver cancer cells (19).

$\mathrm{SPT}$ is a rate-determining enzyme that metabolizes and synthesizes membrane sphingolipids (20). CER, sphingosine and sphingosine-1-phosphate, which are metabolites of sphingolipids, act as intra- and intercellular lipid mediators and are involved in cell proliferation, differentiation and apoptosis $(21,22)$.

As regards the association between NASH and sphingolipids, non-diabetic obese patients with a fatty liver have higher concentrations of CER and sphingomyelin (which is a type of sphingolipid), in their adipose tissue, compared with patients 
Table III. Factors associated with HCC in patients with NAFLD.

\begin{tabular}{|c|c|c|c|c|}
\hline \multirow[b]{2}{*}{ Variables } & \multirow{2}{*}{$\begin{array}{c}\text { Univariate analysis } \\
\text { P-value }\end{array}$} & \multicolumn{3}{|c|}{ Multivariate analysis } \\
\hline & & Odds ratio & $95 \% \mathrm{CI}$ & P-value ${ }^{b}$ \\
\hline Sex & $<0.001$ & 1.008 & $0.890-1.141$ & 0.905 \\
\hline Platelet count & $<0.001$ & 0.629 & $0.415-0.953$ & 0.029 \\
\hline Alanine aminotransferase & $<0.001$ & 0.987 & $0.974-1.000$ & 0.045 \\
\hline Albumin & $<0.001$ & 0.008 & $0.000-0.793$ & 0.040 \\
\hline Triglyceride & 0.037 & 0.990 & $0.968-1.012$ & 0.358 \\
\hline LDL cholesterol & 0.003 & 1.046 & $0.987-1.108$ & 0.133 \\
\hline Hyaluronic acid level & $<0.001$ & 0.993 & $0.985-1.000$ & 0.051 \\
\hline $\mathrm{HbA1c}$ & 0.043 & 1.986 & $0.961-4.163$ & 0.064 \\
\hline$\alpha$-fetoprotein & $<0.001$ & 1.620 & $0.990-2.652$ & 0.055 \\
\hline $\mathrm{DCP}$ & $<0.001$ & 1.001 & $0.993-1.010$ & 0.754 \\
\hline SPTLC3 & 0.001 & 12.935 & $1.002-167.016$ & 0.050 \\
\hline
\end{tabular}

Statistical analyses were performed using ${ }^{a}$ Mann-Whitney U test and ${ }^{b}$ Logistic regression analysis. 95\% CI, 95\% confidence interval; ALT, alanine aminotransferase; LDL, low-density lipoprotein; DCP, des- $\gamma$-carboxy prothrombin; SPTLC3, serine palmitoyltransferase long chain subunit 3 .
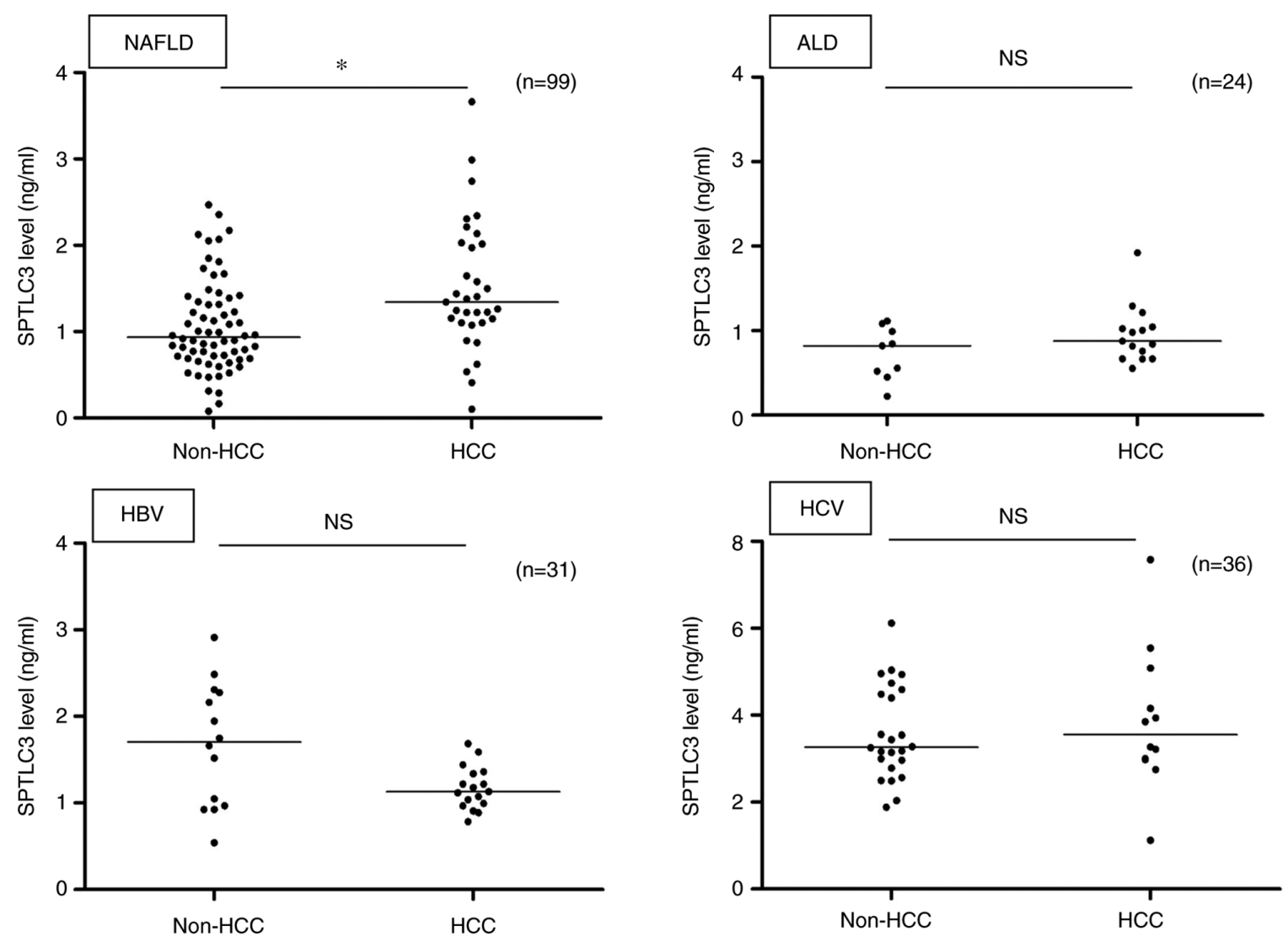

Figure 5. Serum SPTLC3 levels of patients with liver disease of different etiologies without HCC vs. with HCC. The horizontal lines in the dot plot represents the medians. Statistical analyses were performed using the Mann-Whitney U test. ${ }^{*} \mathrm{P}=0.001$, non-HCC vs. HCC. SPTLC3, serine palmitoyltransferase long chain subunit 3; NAFLD, non-alcoholic fatty liver disease; ALD, alcoholic liver disease; HBV, hepatitis B virus; HCV hepatitis C virus.

with healthy livers (23). It has also been reported that hepatic CER levels were increased in mice fed a high-fat diet, and that this elevation was suppressed by the administration of an insulin sensitizer or SPT inhibitor (24). As mentioned above, sphingolipids and their metabolite, ceramide, are implicated in the development of fatty liver. Therefore, it was hypothesized that SPTLC3, which was examined in the present study, is also associated with the progression of NASH pathology. 
Previous reports have indicated an association between SPT and carcinogenesis in malignant melanomas. SPT inhibitors have been shown to attenuate carcinogenesis by inhibiting the $\mathrm{G}_{2} / \mathrm{M}$ transition of malignant melanoma cells (25) and have been reported to suppress the progression of tumors by inhibiting the de novo synthesis of sphingolipids in a mouse model of malignant melanoma (26).

However, there are few reports on the association between SPT and liver cancer. It has been determined that SPT inhibition promotes melatonin-induced apoptosis of HepG2 cells (27); however, to the best of the authors' knowledge, no studies prior to the present study investigated the role of SPTLC3 in human liver cancer.

The non-cancerous sections of the liver in patients with NAFLD expressed significantly higher SPTLC3 mRNA levels compared with the cancerous sections. By contrast, the serum SPTLC3 levels were significantly higher in patients with liver cancer and NAFLD compared with those in patients with NAFLD but without liver cancer. In our previous study, SPTLC3 mRNA levels in non-cancerous liver tissue in a mouse model of NAFLD carcinogenesis were found to increase over time as NASH and carcinogenesis progressed (8). It was hypothesized that, rather than being expressed by cancerous hepatocytes, SPTLC3 may be expressed by normal hepatocytes that have the potential to undergo lipotoxicity and become cancerous, which may be supported by the increase in serum SPTLC3 levels in patients with liver cancer and NAFLD.

Progression of hepatic fibrosis is one of the factors determining the prognosis of patients with NASH $(7,28)$, and it is the most important factor in the pathological progression of NASH (29). In the present study, serum SPTLC3 levels were found to be significantly associated with known liver fibrosis markers, including platelet count, hyaluronic acid levels and FIB-4 index, and with NASH pathogenesis (liver fibrosis progression). This suggests that serum SPTLC3 levels may also be associated with the prognosis of patients with NASH.

AFP and DCP are well-known tumor markers of existing liver cancer; however, AFP levels are not increased in patients with NAFLD-related liver carcinogenesis. In a comparative study of 34 cases of NASH-related liver cancer and 56 cases of HCV-related liver cancer in Japan, the mean values of AFP were 7.0 and $24.0 \mathrm{ng} / \mathrm{ml}$, respectively $(\mathrm{P}=0.007)(30)$. In addition, the level of DCP may increase due to vitamin $\mathrm{K}$ deficiency and, thus, false positives may be associated with malnutrition, oral administration of warfarin, suppression of vitamin production by intestinal bacteria upon oral administration of antibiotics, and impaired absorption of fat-soluble vitamins owing to obstructive jaundice. Therefore, patient medical history should always be taken into consideration.

In the present study, serum SPTLC3 levels were significantly elevated in patients with NAFLD-related HCC, while they remained unchanged in patients with liver diseases of different etiologies (HBV, HCV and alcoholic liver injury). In these groups, no significant increases were observed compared with the non-HCC group, suggesting that SPTLC3 may be a specific tumor marker in patients with NAFLD.

There were two main limitations of the present study. The first limitation was the selection bias of patients. The selected patients with NAFLD with or without HCC were individuals admitted to a medical institution specializing in liver diseases for the purpose of detailed examination and treatment and were not randomly selected from the general population. In the future, it would be beneficial to conduct validation studies in a sample that is larger and more representative of the general population. The second was the insufficient evaluation of SPTLC expression. In the present study, western blotting was not performed to evaluate the level of SPTLC3 protein in the liver cancer tissues. It is necessary to evaluate the protein content in the liver tissues in order to evaluate how SPTLC3 protein expression is reflected in serum SPTLC3 levels, and this will be further studied in the future.

In conclusion, SPTLC3 levels were specifically increased in the serum of patients with NAFLD and HCC, suggesting that SPTLC3 may be involved in liver carcinogenesis in these patients. These findings may highlight the clinical significance of SPTLC3 in the pathogenesis of NAFLD.

\section{Acknowledgements}

The authors would like to thank Dr Nobuhiro Hiraishi, Ms. Hiromi Eguchi and Ms. Yuko Morinaga for their technical assistance.

\section{Funding}

No funding was received.

\section{Availability of data and materials}

The datasets used and/or analyzed during the current study are available from the corresponding authors upon reasonable request.

\section{Authors' contributions}

SI, KO and AI designed the study. OT, AT, HS and KT collected the clinical samples. SI and NH performed reverse transcription-quantitative PCR. SI, KO and SM drafted and revised the manuscript. SI, KK, SK, TT, AM and HU performed statistical and clinical analyses for this study. KO and KT confirm the authenticity of all the raw data. All authors have read and approved the final manuscript.

\section{Ethics approval and consent to participate}

The present study was approved by the Ethics Committee of Kagoshima University Hospital (approval no. 28-107). Written informed consent was obtained from all patients. All procedures were performed according to the World Medical Association's Declaration of Helsinki.

\section{Patient consent for publication}

Not applicable.

\section{Competing interests}

The authors declare that they have no competing interests. 


\section{References}

1. Eslam M, Sanyal AJ and George J, International Consensus Panel: MAFLD: A consensus-driven proposed nomenclature for metabolic associated fatty liver disease. Gastroenterology 58: 1999-2014. e1, 2020.

2. Younossi ZM, Koenig AB, Abdelatif D, Fazel Y, Henry L and Wymer M: Global epidemiology of nonalcoholic fatty liver disease-Meta-analytic assessment of prevalence, incidence, and outcomes. Hepatology 64: 73-84, 2016.

3. Estes C, Anstee QM, Arias-Loste MT, Bantel H, Bellentani S, Caballeria J, Colombo M, Craxi A, Crespo J, Day CP, et al: Modeling NAFLD disease burden in China, France, Germany, Italy, Japan, Spain, United Kingdom, and United States for the period 2016-2030. J Hepatol 69: 896-904, 2018.

4. Oda K, Uto H, Mawatari S and Ido A: Clinical features of hepatocellular carcinoma associated with nonalcoholic fatty liver disease: A review of human studies. Clin J Gastroenterol 8: 1-9, 2015.

5. Tateishi R, Uchino K, Fujiwara N, Takehara T, Okanoue T, Seike M, Yoshiji H, Yatsuhashi H, Shimizu M, Torimura T, et al: A nationwide survey on non-B, non-C hepatocellular carcinoma in Japan: 2011-2015 update. J Gastroenterol 54: 367-376, 2019.

6. Dulai PS, Singh S, Patel J, Soni M, Prokop LJ, Younossi Z, Sebastiani G, Ekstedt M, Hagstrom H, Nasr P, et al: Increased risk of mortality by fibrosis stage in nonalcoholic fatty liver disease: Systematic review and meta-analysis. Hepatology 65: $1557-1565,2017$.

7. Angulo P, Kleiner DE, Dam-Larsen S, Adams LA, Bjornsson ES, Charatcharoenwitthaya P, Mills PR, Keach JC, Lafferty HD, Stahler A, et al: Liver fibrosis, but no other histologic features, is associated with long-term outcomes of patients with nonalcoholic fatty liver disease. Gastroenterology 149: 389-397. e10, 2015.

8. Yoshimine $\mathrm{Y}$, Uto $\mathrm{H}$, Kumagai K, Mawatari S, Arima S, Ibusuki R, Mera K, Nosaki T, Kanmura S, Numata M, et al: Hepatic expression of the Sptlc3 subunit of serine palmitoyltransferase is associated with the development of hepatocellular carcinoma in a mouse model of nonalcoholic steatohepatitis. Oncol Rep 33: 1657-1666, 2015.

9. Chalasani N, Younossi Z, Lavine JE, Charlton M, Cusi K, Rinella M, Harrison SA, Brunt EM and Sanyal AJ: The diagnosis and management of nonalcoholic fatty liver disease: Practice guidance from the American association for the study of liver diseases. Hepatology 67: 328-357, 2018.

10. European Association for the Study of the Liver (EASL); European Association for the Study of Diabetes (EASD); European Association for the Study of Obesity (EASO) EASL-EASD-EASO clinical practice guidelines for the management of non-alcoholic fatty liver disease. J Hepatol 64 1388-1402, 2016.

11. Sterling RK, Lissen E, Clumeck N, Sola R, Correa MC, Montaner J, S Sulkowski M, Torriani FJ, Dieterich DT, Thomas DL, et al: Development of a simple noninvasive index to predict significant fibrosis in patients with $\mathrm{HIV} / \mathrm{HCV}$ coinfection. Hepatology 43: 1317-1325, 2006.

12. Sumida Y, Yoneda M, Hyogo H, Itoh Y, Ono M, Fujii H, Eguchi Y, Suzuki Y, Aoki N, Kanemasa K, et al: Validation of the FIB4 index in a Japanese nonalcoholic fatty liver disease population. BMC Gastroenterol 12: 2, 2012.

13. Lee J, Vali Y, Boursier J, Spijker R, Anstee QM, Bossuyt PM and Zafarmand MH: Prognostic accuracy of FIB-4, NAFLD fibrosis score and APRI for NAFLD-related events: A systematic review. Liver Int 41: 261-270, 2021.

14. Kruger FC, Daniels CR, Kidd M, Swart G, Brundyn K, Van Rensburg C and Kotze M: APRI: A simple bedside marker for advanced fibrosis that can avoid liver biopsy in patients with NAFLD/NASH. S Afr Med J 101: 477-480, 2011.

15. Livak KJ and Schmittgen TD: Analysis of relative gene expression data using real-time quantitative PCR and the 2(-Delta Delta C(T)) method. Methods 25: 402-408, 2001.
16. Nishikoba N, Kumagai K, Kanmura S, Nakamura Y, Ono M, Eguchi H, Kamibayashiyama T, Oda K, Mawatari S, Tanoue S, et al: HGF-MET signaling shifts M1 macrophages toward an M2-like phenotype through PI3K-mediated induction of Arginase-1 Expression. Front Immunol 11: 2135, 2020.

17. Tabassum R, Rämö JT, Ripatti P, Koskela JT, Kurki M, Karjalainen J, Palta P, Hassan S, Nunez-Fontarnau J, Kiiskinen TTJ, et al: Genetic architecture of human plasma lipidome and its link to cardiovascular disease. Nat Commun 10: 4329, 2019.

18. Hornemann T, Wei Y and von Eckardstein A: Is the mammalian serine palmitoyltransferase a high-molecular-mass complex? Biochem J 405: 157-164, 2007.

19. Hornemann T, Richard S, Rütti MF, Wei Y and von Eckardstein A: Cloning and initial characterization of a new subunit for mammalian serine-palmitoyltransferase. J Biol Chem 281: 37275-37281, 2006.

20. Menaldino DS, Bushnev A, Sun A, Liotta DC, Symolon H, Desai K, Dillehay DL, Peng Q, Wang E, Allegood J, et al: Sphingoid bases and de novo ceramide synthesis: Enzymes involved, pharmacology and mechanisms of action. Pharmacol Res 47: 373-381, 2003.

21. Zheng W, Kollmeyer J, Symolon H, Momin A, Munter E, Wang E, Kelly S, Allegood JC, Liu Y, Peng Q, et al: Ceramides and other bioactive sphingolipid backbones in health and disease: Lipidomic analysis, metabolism and roles in membrane structure, dynamics, signaling and autophagy. Biochim Biophys Acta 1758: 1864-1884, 2006

22. Gomez-Larrauri A, Presa N, Dominguez-Herrera A, Ouro A, Trueba M and Gomez-Muñoz A: Role of bioactive sphingolipids in physiology and pathology. Essays Biochem 64: 579-589, 2020.

23. Kolak M, Westerbacka J, Velagapudi VR, Wågsäter D, Yetukuri L, Makkonen J, Rissanen A, Häkkinen AM, Lindell M, Bergholm R, et al: Adipose tissue inflammation and increased ceramide content characterize subjects with high liver fat content independent of obesity. Diabetes 56: 1960-1968, 2007.

24. Cinar R, Godlewski G, Liu J, Tam J, Jourdan T, Mukhopadhyay B, Harvey-White $\mathrm{J}$ and Kunos G: Hepatic cannabinoid-1 receptors mediate diet-induced insulin resistance by increasing de novo synthesis of long-chain ceramides. Hepatology 59: 143-153, 2014.

25. Lee YS, Choi KM, Choi MH, Ji SY, Lee S, Sin DM, Oh KW, Lee YM, Hong JT, Yun YP, et al: Serine palmitoyltransferase inhibitor myriocin induces growth inhibition of B16F10 melanoma cells through G(2)/M phase arrest. Cell Prolif 44: 320-329, 2011.

26. Lee YS, Choi KM, Lee S, Sin DM, Lim Y, Lee YM, Hong JT, Yun YP and Yoo HS: Myriocin, a serine palmitoyltransferase inhibitor, suppresses tumor growth in a murine melanoma model by inhibiting de novo sphingolipid synthesis. Cancer Biol Ther 13: 92-100, 2012.

27. Ordoñez R, Fernández A, Prieto-Domínguez N, Martínez L, García-Ruiz C, Fernández-Checa JC, Mauriz JL and González-Gallego J: Ceramide metabolism regulates autophagy and apoptotic cell death induced by melatonin in liver cancer cells. J Pineal Res 59: 178-189, 2015.

28. Hagström H, Nasr P, Ekstedt M, Hammar U, Stål P, Hultcrantz R and Kechagias S: Fibrosis stage but not NASH predicts mortality and time to development of severe liver disease in biopsy-proven NAFLD. J Hepatol 67: 1265-1273, 2017.

29. Loomba R and Chalasani N: The hierarchical model of NAFLD: Prognostic significance of histologic features in NASH. Gastroenterology 149: 278-281, 2015.

30. Tokushige K, Hashimoto E, Yatsuji S, Tobari M, Taniai M, Torii N and Shiratori K: Prospective study of hepatocellular carcinoma in nonalcoholic steatohepatitis in comparison with hepatocellular carcinoma caused by chronic hepatitis C. J Gastroenterol 45: 960-967, 2010.

This work is licensed under a Creative Commons Attribution-NonCommercial-NoDerivatives 4.0 International (CC BY-NC-ND 4.0) License. 